\title{
Characteristics of Viral Shedding in Respiratory Samples and Specific Antibodies Production in 564 COVID-19 Patients
}

\author{
Jing GONG ${ }^{1}$, Hui DONG ${ }^{2}$, Ding-kun $\mathrm{WANG}^{1}$, Fu-er $\mathrm{LU}^{2}$, Zhao-yi HUANG ${ }^{1}, \mathrm{Ke} \mathrm{FANG}^{1}$, Wen-ya HUANG ${ }^{1}$, Fen YUAN ${ }^{1}$, \\ Xing $\mathrm{CHEN}^{3}$, Qing-song XIA², Le-yi $\mathrm{MA}^{2}$, Fan $\mathrm{WU}^{2}$, Hao $\mathrm{SU}^{2}$, Min-min $\mathrm{GONG}^{2}$, Yue-heng TANG ${ }^{2}$, Ke-xin NIE², \\ Zhi WANG ${ }^{2}$, Sheng-hao TU' ${ }^{1}$, Ming-min ZHANG ${ }^{1}$, Jing-bin LI ${ }^{1 \#}$ \\ ${ }^{1}$ Department of Integrated Traditional Chinese and Western Medicine, Tongji Hospital, Tongji Medical College, Huazhong \\ University of Science and Technology, Wuhan 430030, China \\ ${ }^{2}$ Institute of Integrated Traditional Chinese and Western Medicine, Tongji Hospital, Tongji Medical College, Huazhong \\ University of Science and Technology, Wuhan 430030, China \\ ${ }^{3}$ Department of Clinical Laboratory, Tongji Hospital, Tongji Medical College, Huazhong University of Science and Technology, \\ Wuhan 430030, China
}

(C) Huazhong University of Science and Technology 2021

\begin{abstract}
Summary: Positive nucleic acid (NA) results have been found in recovered and discharged COVID-19 patients, but the proportion is unclear. This study was designed to analyze the recurrent positive rate of NA results after consecutively negative results, and the relationship between the specific antibody production and positive NA rate. According to Strengthening the Reporting of Observational Studies in Epidemiology guidelines, data of inpatients in Sino-French New City Branch of Tongji Hospital between Jan. 28 and Mar. 6, 2020 were collected. A total of 564 COVID-19 patients over 14 years old who received the examinations of NA and antibodies against SARS-CoV-2 were included. Days of viral shedding and specific antibodies were recorded and assessed. Among NA tests in respiratory samples (throat swabs, nasopharyngeal swabs, sputum and flushing fluid in alveoli), the patients with all-negative NA results accounted for $17.20 \%$, those with single-positive results for $46.63 \%$, and those with multiple-positive results for $36.17 \%$ respectively. Besides, the recurrent positive NA results after consecutively negative results appeared in 66 patients $(11.70 \%)$. For multiple-positive patients, median viral shedding duration was 20 days (range: 1 to 57 days). Of the 205 patients who received 2 or more antibody tests, $141(68.78 \%)$ had decreased $\operatorname{IgG}$ and $\operatorname{IgM}$ concentrations. IgM decreased to normal range in 24 patients, with a median of 44 days from symptom onset. Viral shedding duration was not significantly correlated with gender, age, disease severity, changes in pulmonary imaging, and antibody concentration. It is concluded that antibody level and antibody change had no significant correlation with the positive rate of NA tests and the conversion rate after continuous negative NA tests. In order to reduce the recurrent positive proportion after discharge, 3 or more consecutive negative NA test results with test interval more than $24 \mathrm{~h}$ every time are suggested for the discharge or release from quarantine.

Key words: COVID-19; viral shedding; specific antibodies
\end{abstract}

The global outbreak of COVID-19 poses serious health threat ${ }^{[1]}$. Figuring out the characteristics of viral shedding and antibody against COVID-19 plays key roles for international epidemic control. The positive real time-polymerase chain reaction (RTPCR) results of nucleic acid (NA) were reported in recovered and discharged COVID-19 patients $^{[2]}$. However, the proportion of recurrent positive NA results after consecutively negative results is not clear. Antibody test of COVID-19 patients has played an important role in $\mathrm{China}^{[3-5]}$. It is still not completely clear whether the specific antibodies could decrease

Jing GONG, E-mail: jgongtcm@126.com

"Corresponding author, E-mail: tjlaojing@163.com viral shedding. This study retrospectively analyzed the clinical characteristics of NA test results and antibody production in inpatients to explore the rate of recurrent positive NA results after consecutively negative results, and the relationship between the specific antibody production and positive NA rate.

\section{MATERIALS AND METHODS}

\subsection{Study Design}

In this retrospective cohort study, Strengthening the Reporting of Observational Studies in Epidemiology (STROBE) guidelines were used for study design and clinical data collection. In Sino-French New City Branch of Tongji Hospital, a designated hospital for 
COVID-19, the data of inpatients from Jan. 28, 2020 to Mar. 6, 2020 were collected. Data collection was completed on Mar. 20, 2020. The inclusion criteria were as follows: (1) above 14 years of age; (2) both NA and antibody against COVID-19 were tested; (3) positive NA test result, or negative NA with increased IgM or IgG concentration; (4) diagnosis of viral pneumonia by imaging examinations.

This study was approved by the Ethical Committee of Tongji Hospital, Tongji Medical College, Huazhong University of Science and Technology. Informed consent was waived by the ethical commission. All data were anonymous before use.

\subsection{Data Collection}

From electronic medical records, we collected the information about age, gender, symptom onset time, NA test results in respiratory samples (throat swabs, nasopharyngeal swabs, sputum and flushing fluid in alveoli) before and during hospitalization, antibody test results, disease severity, and $\mathrm{CT}$ and chest radiography changes. For patients who received multiple NA tests in respiratory samples within $24 \mathrm{~h}$, a positive result at least once on a NA test was deemed positive; otherwise, negative NA result was deemed. The NA test results before admission to this hospital were obtained from medical records or telephone consultation.

The disease conditions included common, severe, critical and dead. Referring to "New CoronavirusInfected Pneumonia" Severe and Critical Diagnosis and Treatment Program (second trial version) formulated by the National Health Commission of China, patients with blood oxygen saturation less than $93 \%$ in resting state, or respiratory frequency greater than 30 times/ min were classified as severe cases; those needing mechanical ventilation were deemed as critical cases. Although lots of patients have been recovered and discharged, disease condition was classified according to the most serious conditions.

\subsection{Test Methods}

Pulmonary imaging changes were classified according to the radiologist's reports ${ }^{[6]}$. Reduction or dilution of ground-glass opacities was defined as improvement; little change on range or density of ground-glass opacities was defined as stabilization; otherwise, aggravation was deemed. NAtesting methods have been reported elsewhere ${ }^{[7]}$. Serum concentration of antibody against COVID-19 was measured by the chemiluminescence method. According to NA test and antibody test results, days from symptom onset to first detectable viral shedding, duration from symptom onset to last detectable viral shedding, duration of viral shedding, positive NA rate, rate of recurrent positive NA results after consecutively negative results, and the change trend of IgM and IgG antibody were assessed.

\subsection{Statistical Analysis}

The continuous variables were illustrated by medians, interquartile range (IQR) and ranges. Categorical variables were expressed by count and percentage. Correlation analyses were carried out on the basis of data distribution. Pearson correlation coefficient was used for continuous data, and the Spearman correlation coefficient was used for categorical variables. Data analysis was conducted by SPSS 20.0.

\section{RESULTS}

\subsection{Clinical Characteristics of Included Patients}

Totally, 1457 patients were admitted to out hospital before Mar. 6, 2020, and 856 patients were excluded from this study because specific antibody against COVID-19 was not tested or age was below 14 years. Among 601 patients who received specific antibody testing, 37 patients were excluded due to negative NA results and negative or weak positive antibody against COVID-19 (IgG <20 AU/mL) despite of changes in pulmonary imaging. For 564 COVID-19 patients enrolled, age ranged between 14 and 92 years (table 1 ), and 291 patients $(51.60 \%)$ were female. Regarding disease severity, there were 132 common cases (23.4\%), 340 severe cases $(60.28 \%), 92$ critical cases (16.84\%), and 11 deaths (1.95\%). A total of 513 patients underwent 2 or more chest imaging examinations (X-ray examination for 7 patients and CT examinations for 506) in this hospital. The improvements were found in 436 patients $(86.17 \%)$, the stabilization was found in 53 patients $(9.40 \%)$, and the aggravation occurred in 24 patients $(4.26 \%)$.

\subsection{NA Test Times, Positive NA Rate, NA Test Time from Symptom Onset and Viral Shedding Duration in Patients}

The median NA test times from symptom onset in respiratory samples were 5 (range 2-13) in the included patients. Overall positive NA rate was $25 \%$, and $100 \%$ of positive rate was found in 3 patients with multiple tests. A total of 97 patients (17.20\%) achieved

Table 1 Characteristics of nucleic acids test results among included COVID-19 patients

\begin{tabular}{lcc}
\hline Characteristics & Median (IQR) & Range \\
\hline Age (years) & $64(54-71)$ & $14-92$ \\
First detectable viral shedding (days) & $9(4-13)$ & $0-54$ \\
Viral shedding duration (days) & $20(13.75-29)$ & $1-57$ \\
Last detectable viral shedding (days) & $15.5(8-29)$ & $0-89$ \\
NA test times & $5(4-6)$ & $2-13$ \\
Positive NA rate (\%) & $25(16.67-37.5)$ & $0-100$ \\
\hline
\end{tabular}


all negative results, 263 patients were single-positive (46.63\%), and 204 patients (36.17\%) were multiplepositive. Of the patients with positive NA results, duration data from symptom onset to first detectable viral shedding were extracted in 450 patients, and median duration was 9 days (range $0-54$ ). The median viral shedding duration was 20 days with a maximum of 57 days. Duration data from symptom onset to last detectable viral shedding were available in 460 patients, and the median was 15.5 days (range 1-89 days); besides, the median was 9 days (range 0-51 days) in single-positive patients and 29.5 days (range 6-89 days) in multiple-positive patients.

\subsection{Recurrent Positive NA Results after Consecutively Negative Results}

Recurrent positive NA results after 2 consecutively negative results appeared in 66 patients $(11.70 \%)$, 11 of them were single-positive, and 55 people were multiple-positive. Recurrent positive results after 3, 4, and 5 consecutively negative NA tests happened in 20 patients (3.55\%), 6 patients $(1.06 \%)$, and in one patient $(0.18 \%)$, respectively.

2.4 Testing Range of Antibodies against COVID-19 and Antibody Change Trend in Included Patients

Because 4 patients were positive for NA results and negative for antibody test, 560 patients were included in the analysis of antibody. IgM concentration ranged from 0.33 to $11002.95 \mathrm{AU} / \mathrm{mL}$, and $\mathrm{IgG}$ concentration fluctuated between 8.01 and $1291.17 \mathrm{AU} / \mathrm{mL}$.

A total of 205 patients received 2 or more antibody examinations. Both increases of IgM and IgG happened in 18 patients $(8.78 \%)$, the increase of IgM with the decrease of IgG occurred in 6 patients $(2.93 \%)$, both decreases of $\operatorname{IgM}$ and $\operatorname{IgG}$ occurred in 141 patients $(68.78 \%)$, and the decrease of IgM and the increase of IgG were shown in 40 patients (19.51\%). Decrease of IgM to normal level was found in 24 patients in multiple antibody tests, and the median days from symptoms onset was 44. Fig. 1 shows the median change of IgM or IgG and the percentage of patients with normal or close to normal IgM levels over time. Although $68.78 \%$ patients in multiple antibody tests showed decreased antibodies, there was no significant change in the average antibody levels among all included patients over time because of long-sustained and high antibody level of some patients. One patient had significantly higher IgM levels than those of others, and the IgM concentration was $11002.95 \mathrm{AU} / \mathrm{mL}$ on 45 th day from symptom onset, $9023.03 \mathrm{AU} / \mathrm{mL}$ on 49th day, and $4613.21 \mathrm{AU} / \mathrm{mL}$ on $53 \mathrm{rd}$ day. In a newly-onset patient, IgM and IgG levels were normal $(<10 \mathrm{AU} / \mathrm{mL})$ on 5 th day, $\operatorname{IgM}$ increased to 657.93 and $\operatorname{IgG}$ increased to 123.84 on 15 th day, and antibody level decreased by more than $50 \%$ on the 23 rd day.

\subsection{Analysis of Last NA and Antibody Testing Results}

On the basis of the last $2 \mathrm{NA}$ testing results and the last antibody testing, $\mathrm{NA}^{+} \operatorname{IgM}^{+} \operatorname{IgG}{ }^{+}$was found in 23 cases $(4.08 \%), \mathrm{NA}^{+} \operatorname{IgM}^{-} \operatorname{IgG}^{+}$was found in 11 cases $(1.95 \%), \mathrm{NA}^{-} \operatorname{IgM}{ }^{+} \operatorname{IgG}^{+}$was found in 426 cases (75.53\%), $\mathrm{NA}^{-} \operatorname{IgM}-\operatorname{IgG}^{+}$was found in 100 cases $(17.73 \%)$, and $\mathrm{NA}^{-} \mathrm{IgM}^{-} \mathrm{IgG}^{-}$was found in 4 cases $(0.71 \%)$. In $11 \mathrm{NA}^{+} \operatorname{IgM}-\operatorname{IgG}{ }^{+}$patients, 8 patients received multiple specific antibody tests, and IgG titers decreased.

2.6 Correlation between Positive NA Rate, Viral Shedding Duration or Recurrent Positive NA Results and Other Factors

The correlation analyses revealed that gender, age, disease severity, pulmonary imaging changes, antibody levels, and antibody change were not significantly associated with positive NA rate, recurrent positive NA results and viral shedding duration. Antibodies levels were correlated with disease severity and age (correlation coefficient $<0.2, P<0.05$ ).

\section{DISCUSSION}

Among several viral shedding routes, respiratory samples were most used for NA testing in clinical practice $^{[8]}$. Few positive NA results were reported in anal swabs of adults ${ }^{[9,10]}$. Even if the continuous negative NA testing results in nasopharyngeal swabs, positive NA testing results in anal swabs could be found in several children ${ }^{[11,12]}$. Considering possibility of different NA testing features in children and adults,
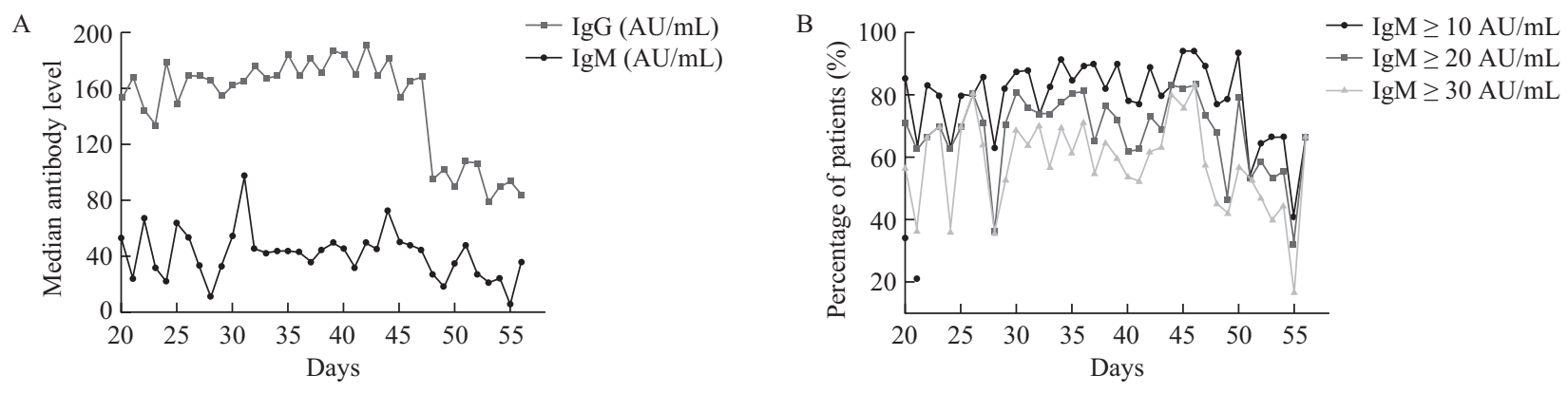

Fig. 1 Changes of antibody against COVID-19 in patients between 20 and 58 days from symptom onset A: median antibody level per day between 20 and 58 days from symptom onset; B: the percentage of patients with IgM antibody against COVID-19 above 10, 20 or $30 \mathrm{AU} / \mathrm{mL}$ per day between 20 and 58 days from symptom onset 
NA testing results in respiratory samples in inpatients over 14 years were included in this study. It has been reported that positive NA testing result was found in discharged patients ${ }^{[2]}$. In ourstudy, although the majority of patients had improved imaging changes and clinical symptoms (discontinuation of ventilator, getting rid of oxygen mask, and so on) ${ }^{[6]}$, the percentage of positive NA testing results after consecutively negative results accounted for $11.70 \%$ in all patients: $3.55 \%, 1.065 \%$ and $0.18 \%$ after 3,4 and 5 consecutive negative tests, respectively. With the increase of the NA test times, the NA positive rate after consecutive negative tests decreased. Three or more consecutive negative results in NA testing with a test interval more than $24 \mathrm{~h}$ every time are suggested for the release from quarantine.

Median day from symptom onset to first detectable viral shedding was 9 , the shortest was 0 , namely NA test was positive on the day of symptom onset, and the longest was 54 . This could be interpreted by limited NA test kit in initial phase and subsequent increase. Similar to a study by Zhou et al, the median viral shedding duration was 20 days $^{[13]}$, while the longest virus shedding period was 57 days in this study.

Positive NA results and normal antibody level were found in 4 of 564 included patients, suggesting antibody sensitivity was over $99 \%$. It cannot be excluded that few COVID-19 patients did not produce specific antibodies. Because of the difference of antibody levels and durations between individuals, no significant decrease trend over time was found on the overal average levels of specific antibodies (fig. 1). According to the last two NA testing results and the last antibody testing results, $93.93 \%$ patients were in the recovery period, and 23 patients $(6.07 \%)$ were during the infection-active period. In $\mathrm{NA}^{+} \operatorname{IgM}-\operatorname{IgG}^{+}$ patients, IgG titers were decreased even with viral shedding, which suggested IgG titers did not parallel with viral shedding. Possibility of false-negative NA testing results could not be completely ruled out in socalled recovery period. On 9th day, normal IgM level and increased IgG levels were found in one patient, indicating that antibodies had been produced in the symptoms-free incubation period in some patients.

Disease severity is considered to be related to the duration of viral shedding ${ }^{[14,15]}$. However, in this study, no significant correlation was found between duration of viral shedding and disease severity (correlation coefficient $=0.012, P=0.746$ ), pulmonary imaging changes (correlation coefficent $=-0.009, P=0.82$ ), and antibody levels (for IgM: correlation coefficent $=0.01, P=0.99$; for IgG: correlation coefficient $=0.044, P=0.348$ ). Many patients were transferred to our hospital in critical period, and lots of critically-ill patients had been improved and discharged from hospital. Antibody test was available from late February, and lack of early onset data on NA testing and antibody levels may be one possible cause.

Antibody levels are significantly related to disease severity, which may be related to immune activation in "cytokine-storm" condition of critical and severe patients $^{[16-18]}$. Decrease of $\operatorname{IgM}$ and IgG levels was found in $68.78 \%$ patients with multiple antibody tests. Epidemiological survey with large sample size helps to find the exact changes of antibodies over time.

Several limitations exist in this retrospective study. Many patients were transferred to our hospital in critical period, and early-onset data may be limited. Some prehospital examination results in several patients were not accessible. A lot of patients received NA tests in different hospitals, and the examination results were affected by sampling of the operators. Few positive NA results were found in anal swabs in adults, only NA results of respiratory tract samples were included. Majority of patients received NA examination using pharynx or nasopharyngeal swabs, sputum or alveolar lavage fluid samples were also included. The NA results from different respiratory samples are affected by different factors, which could lead to some bias. For multiple NA tests within $24 \mathrm{~h}$, only one result was used, which may reduce the positive rate after consecutive negative results. Furthermore, the intervals between NA tests varied. When the antibody test kits were available, most included patients had produced specific antibodies, and the antibody changes during disease onset were not accessible in this study.

In brief, antibody level and antibody change trend had no significant correlation with the positive rate of NA tests and the conversion rate after continuous negative NA testing results. In order to reduce the recurrent positive rate after discharge, 3 or more consecutive negative NA test results with test interval more than $24 \mathrm{~h}$ every time are suggested for the discharge or release from quarantine.

\section{Conflict of Interest Statement}

All authors declare that there are no conflicts of interest.

\section{REFERENCES}

1 Guan WJ, Ni ZY, Hu Y, et al. Clinical Characteristics of Coronavirus Disease 2019 in China. N Engl J Med, 2020,382(18):1708-1720

2 Lan L, Xu D, Ye G, et al. Positive RT-PCR Test Results in Patients Recovered From COVID-19. JAMA, 2020, 323(15):1502-1503

3 Dong X, Cao YY, Lu XX, et al. Eleven Faces of Coronavirus Disease 2019. Allergy, 2020,75(7):16991709

4 Guo L, Ren L, Yang S, et al. Profiling Early Humoral Response to Diagnose Novel Coronavirus Disease (COVID-19). Clin Infect Dis, 2020,71(15):778-785

5 Li Z, Yi Y, Luo X, et al. Development and Clinical Application of A Rapid IgM-IgG Combined Antibody Test for SARS-CoV-2 Infection Diagnosis. J Med Virol, 2020,92(9):1518-1524 
6 Shi H, Han X, Jiang N, et al. Radiological findings from 81 patients with COVID-19 pneumonia in Wuhan, China: a descriptive study. Lancet Infect Dis, 2020,20(4): 425-434

7 Chen T, Wu D, Chen H, et al. Clinical characteristics of 113 deceased patients with coronavirus disease 2019: retrospective study. BMJ, 2020,368:m1091

8 Zhang W, Du RH, Li B, et al. Molecular and serological investigation of 2019-nCoV infected patients: implication of multiple shedding routes. Emerg Microbes Infect, 2020,9(1):386-389

9 Liu Y, Yan LM, Wan L, et al. Viral dynamics in mild and severe cases of COVID-19. Lancet Infect Dis. 2020,20(6):656-657

$10 \mathrm{Wu} \mathrm{J}$, Liu J, Li S, et al. Detection and analysis of nucleic acid in various biological samples of COVID-19 patients. Travel Med Infect Dis, 2020,37:101673

11 Cai J, Xu J, Lin D, et al. A Case Series of children with 2019 novel coronavirus infection: clinical and epidemiological features. Clin Infect Dis, 2020,71(6): 1547-1551

$12 \mathrm{Su} \mathrm{L}$, Ma X, Yu H, et al. The different clinical characteristics of corona virus disease cases between children and their families in China --- the character of children with COVID-19. Emerg Microbes Infect, 2020, 9(1):707-713

13 Zhou F, Yu T, Du R, et al. Clinical course and risk factors for mortality of adult inpatients with COVID-19 in Wuhan, China: a retrospective cohort study. Lancet, 2020,395(10229):1054-1062

$14 \mathrm{Xu} \mathrm{K}$, Chen Y, Yuan J, et al. Factors Associated With Prolonged Viral RNA Shedding in Patients with Coronavirus Disease 2019 (COVID-19). Clin Infect Dis, 2020,71(15):799-806

15 Zhang W, Du RH, Li B, et al. Molecular and serological investigation of 2019-nCoV infected patients: implication of multiple shedding routes. Emerg Microbes Infect, 2020,9(1):386-389

$16 \mathrm{Hu} \mathrm{B}$, Huang S, Yin L. The cytokine storm and COVID-19. J Med Virol, 2020,10.1002/jmv.26232

17 Nile SH, Nile A, Qiu J, et al. COVID-19: Pathogenesis, cytokine storm and therapeutic potential of interferons. Cytokine Growth Factor Rev, 2020,53:66-70

18 Ye Q, Wang B, Mao J. The pathogenesis and treatment of the "Cytokine Storm" in COVID-19. J Infect, 2020, 80(6):607-613

(Received May 21, 2020; accepted Oct. 28, 2020) 